\title{
The use of frequency converters on three-circuit ferroresonant circuits
}

\author{
Bobokul Shaymatov ${ }^{*}$, Maktuba Rakhmatova, Sardor Komilov, Dilnoza Qurbonova \\ Bukhara Engineering-Technological Institute, Bukhara, Uzbekistan
}

\begin{abstract}
In this paper, the processes of formation of low-frequency currents in the circuits of ferromagnetic oscillations are analyzed, and the theory of the formation of secondary amplitude frequencies of soft excitation of parallel and series-connected ferroresonant chains is developed. Based on this, considering the use of frequency dividers in the irrigation system shown in Figure 1, it is possible to carry out experiments on the study of multi-circuit frequency dividers. Therefore, the study of spontaneous (soft) vibration processes in multi-circuit ferroresonant electric circuits of periodic changes in the parameters of a ferromagnetic element generated by the resulting harmonic oscillations (HA) and their development and application of improved sources of harmonic signals for telecontrol of the system in power lines, switchgears and drainage networks.
\end{abstract}

\section{Introduction}

The study of multi-circuit ferroresonance circuits is related, firstly, to some generalizations about self-oscillating processes in multi-circuit circuits, and secondly, to the need to further improve telesignaling systems (TS) along power transmission lines (APT) in regional distribution networks. In this case, the main element of ferroresonance chains is used as a low-harmonic frequency divider.

At present, the analyzes show that single and multi-circuit ferroresonance circuits are fully described, but the theory and design of the devices are not yet complete with the use of low-power transformers for these circuits. The task of studying the properties of different types of parametric systems and creating a methodology for determining their feasibility in science and technology is urgent. Accordingly, the need for converters of various types and functional purposes is mainly realized based on semiconductor devices. At present, a telesignal system for regional distribution networks, including reclamation networks of vertical drainage based on ferroresonance converters, has been created.

Ensuring full electromagnetic compatibility of secondary sources with the supply network while increasing the quality and reliability of the transmitted electricity leads to the complication of control systems. Therefore, using a telecontrol system for the modernization of reclamation electrical networks based on semiconductor elements of microcircuits presents certain difficulties. The main thing, in principle, is that the device in

\footnotetext{
* Corresponding author: boboqul.shaymatov@mail.ru
} 
which the semiconductors are located cannot create the required temperature regime under the influence of the external environment.

For this purpose, multicircuit ferroresonance is the study of spontaneous (soft) vibration processes in electrical circuits and the creation on their basis of improved sources of harmonic signals for telecontrol systems in power lines, switchgear and drainage networks.

This is achieved by solving the following specific tasks:

- Theoretical and experimental studies of frequency dividers with low harmonic oscillations at output frequencies of $162 / 3 \mathrm{~Hz}$ and $25 \mathrm{~Hz}$;

- to achieve smooth excitation of the tertiary harmonic frequencies at the output terminals of the frequency divider by choosing the parameters and structure of the circuit;

- widening the range of low frequencies of harmonics and optimization of the electromagnetic regime;

- development of new principles for coordinating the signaling system with the highvoltage network;

Develop telecontrol systems for vertical drainage pumps adapted for use in reclamation electrical networks $[5,7,8,11,12,13,16]$.

\section{Methods}

In this regard, the task of developing a methodology for studying the properties of various types of parametric systems and determining their feasibility in science and technology is urgent. It is also planned to improve the collector and drainage network in the melioration and irrigation system using "soft" excitation of harmonic frequencies. These tasks, in turn, exacerbate the problem of monitoring the condition of vertical drainage pumps. It should be noted that in the Bukhara region alone, there are more than 1000 vertical drainage structures designed to improve land reclamation.

Typically, vertical drainage pumps are located within a radius of $15-20 \mathrm{~km}$. Of course, this raises the question of effective pump control, constant monitoring of information about their operating condition. As a result, drainage pumps can be left unattended for a long time due to emergencies (accidents). Therefore, today it is relevant to develop technical conditions and technical systems that allow you to have constantly up-to-date information on the state of vertical drainage pumps during the day.

The need to equip distribution networks with technical equipment is obvious. However, 6.10 and $35 \mathrm{kV}$ distribution networks do not have telecontrol and remote signaling systems that meet all the generally accepted requirements for such systems. High-frequency communication in distribution networks via power lines in networks with a voltage of 110 $\mathrm{kV}$ and above is difficult since the use of high-frequency communication creates an extreme problem. As a result, it becomes necessary to use the technical control and remote signaling systems in general, which are widely used in distribution networks.

In view of the above, at present, it is important to provide exploitation Discount data substations $6,10 / 0.4 \mathrm{kV}$ and $35 / 6 / 0.4 \mathrm{kV}$ distribution networks $6,10,35$ and $110 \mathrm{kV}$ and their connection to the control centers.

Transmission and reception of television signals are carried out at low harmonic frequencies and have significant advantages $[5,7,16,20]$.

Today, it is important to realize the most efficient means of data transmission over the power lines of low-voltage distribution networks through telecontrol and TV signals.

Such means of communication can significantly reduce damage in the event of failure of vertical drainage pumps in reclamation networks, for example, due to the timely management of compensation devices. This is very important since many pumping stations are switching to high-tech equipment of foreign design. 
At present, telecontrol and telesignaling systems use ultraharmonic frequencies of 100 $3000 \mathrm{~Hz}$ and subharmonic frequencies of $25 \mathrm{~Hz}, 162 / 3 \mathrm{~Hz}$, and below.

The choice of frequency is determined by the following key factors:

- the dependence of the voltage losses of the frequency signal in different sections of the distribution networks;

- the degree of interference of signals of different frequencies with signals during destructive modes in the distribution network.

The choice of signal generation method is decided by determining the basic requirements for a given situation. We offer to use ferroresonant frequency dividers as one of the methods for generating an active signal. Such frequency dividers are simple in appearance, can withstand very high loads, operate reliably at high ambient temperatures, and therefore are convenient for use in telecontrol and remote signaling systems in hot climates $[1,2,10,16]$.

The circuit shown in Figure 1 is considered basic in the manufacture and use of ferroresonant frequency dividers.

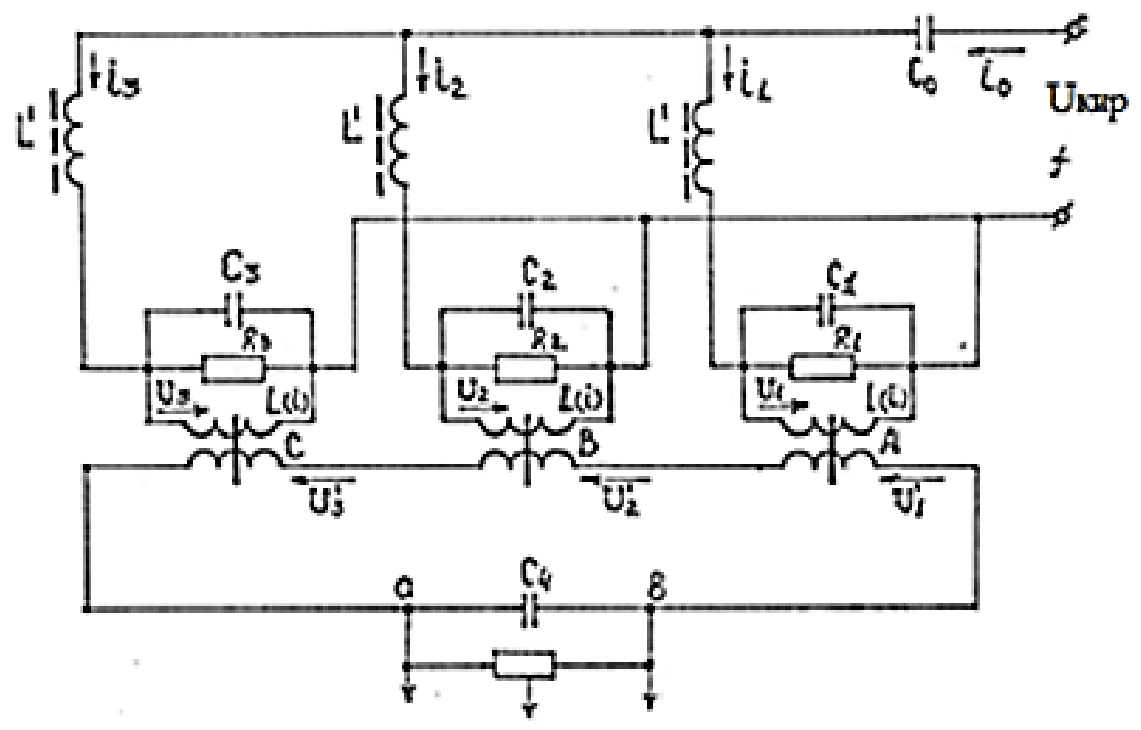

Fig. 1. Ferroresonant frequency divider

Analyzing the specific characteristics of the excitation of low harmonic frequencies (LHF) at the input of nonlinear processes and ferroresonance chains, we obtain frequencies at $162 /$ (3) $\mathrm{Hz}$ and $25 \mathrm{~Hz}$, different from the base frequency, creating a "soft" excitation at the output of the frequency divider.

In an experimental study of this scheme, when analyzing Fig. 2, 3 modes of operation of the oscillating circuit were observed, which are described by the curve of the dependence of the input voltage on the current in the primary circuit. With a smooth increase in the input voltage in the "curve" of the "ab" part of the Weber-ampere characteristic, the selfoscillation mode is unstable. 


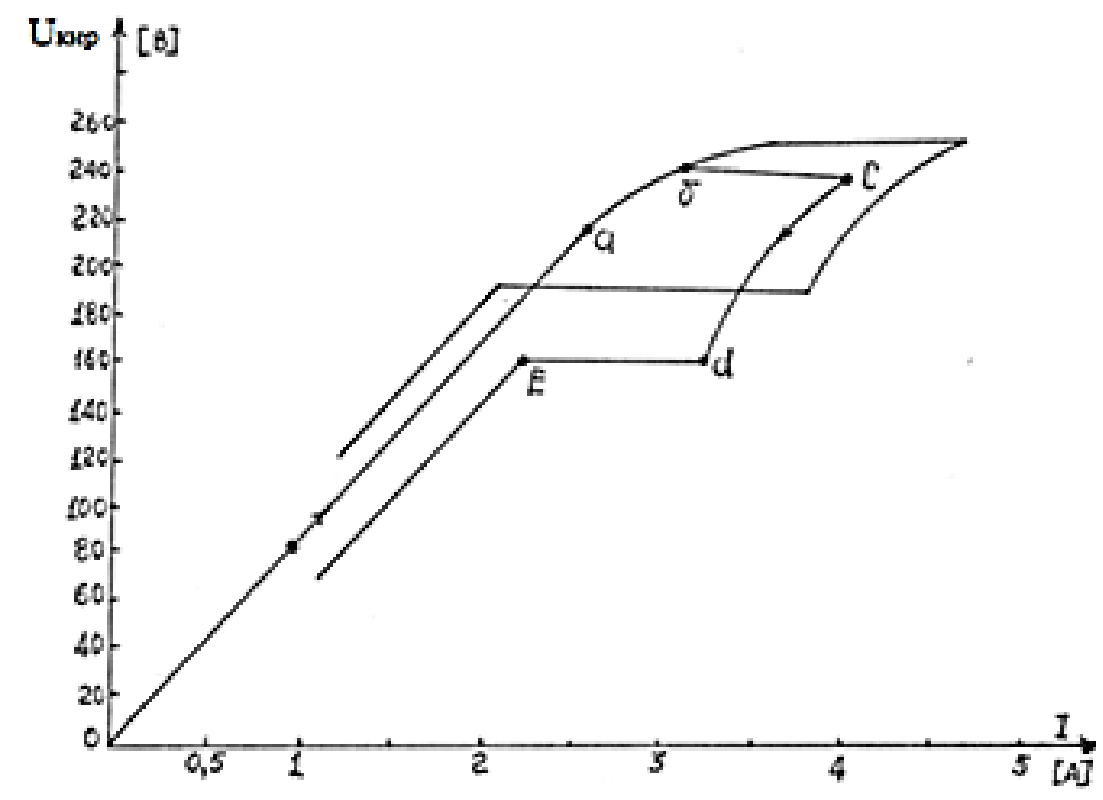

Fig: 2. Weber-ampere characteristic.

In addition, with a flat increase in the voltage at the input of the ferroresonant circuit, the current "bc" sharply increases, and a stable mode of formation of secondary FFD is established $[6,7]$.

The proposed ferroresonant frequency divider ( FFD ) is a type of filter that has the form of the main frequency components of the mains voltage. The presence of single-phase voltage with frequency at the output F of PM is different from the network frequency coding signals that allow the passage of the frequency phase and generate four different signals with a frequency of 180 different power levels $\frac{\omega}{2}$ and $\frac{\omega}{3} \mathrm{~Hz}$. The block diagram of remote control and remote signaling is shown in Figure 3 and consists of two parts:

- Transmitter ASB, command block (KB), and Consists of a voltage transformer of type VT (VT-2).

- the receiving device includes the following :

Voltage transformer VT (VT-1);

frequency filter ( FF ) signal selector block containing (SSB );

phase-sensitive (PS ) device; local harmonic oscillator ( LHO ); GHO unit, consisting of three relays (R); executive mechanisms (EM).

Depending on the commands coming from the means of relay protection and automatic connection, the KB ASB includes the frequency of the phase voltage T $\mathrm{c}$. The set voltage generates a current in the coils equilibrium VT 1, which is part of the zero sequence, and therefore amplifies the signal from PMVT-2, which is tuned to the resonance frequency T c to VT-2. In this case, the signal switch unit issues a command to the actuator via the appropriate relay, depending on the phase of the incoming signal. 


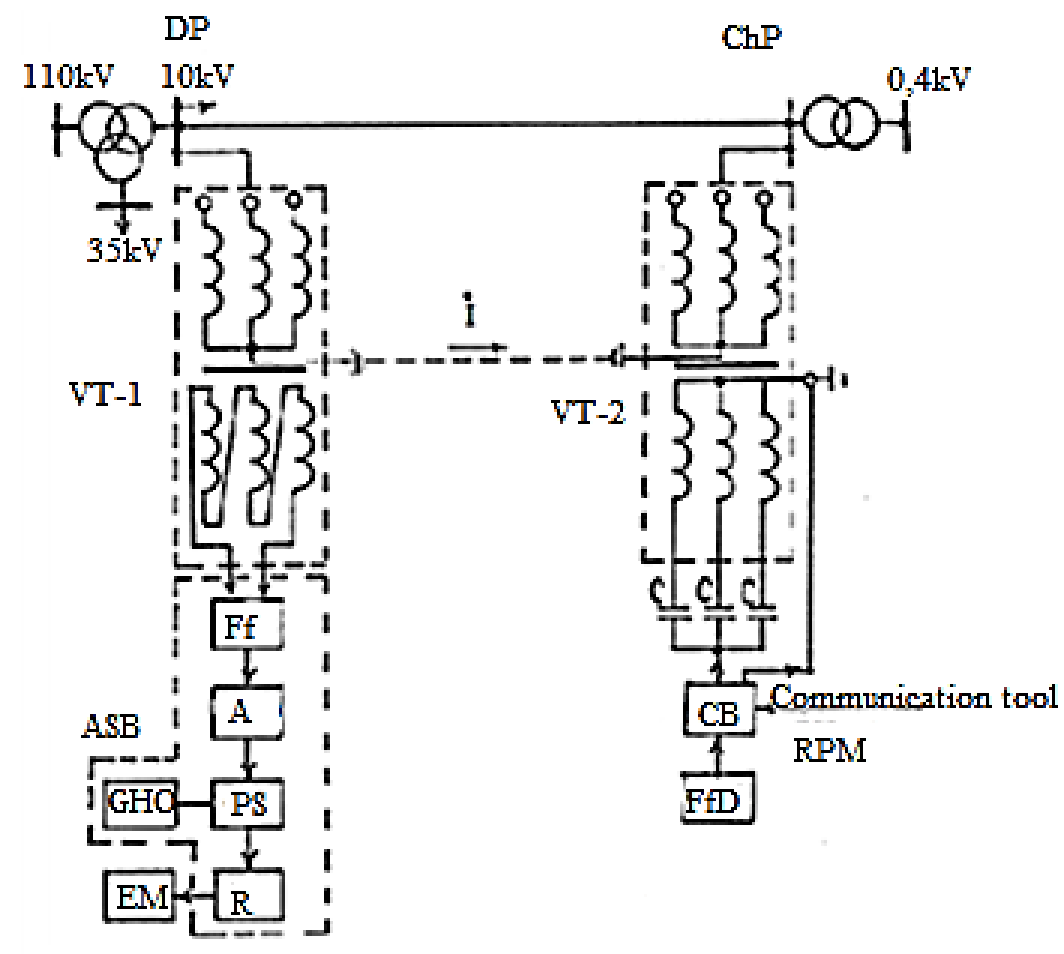

Fig.3. Block diagram of telecontrol and remote signaling.

Depending on the neutral mode of the transformers and the presence of VT (or VT-35) type VT-2 at the substation, the input signal power can be realized through the secondary windings of the transformers. Suppose a signal or command is transmitted at the substation control point, that is, from an existing voltage transformer model VTMI. In that case, the signal is transmitted through secondary windings connected to the "star." In this case, the ground in the ASB transformer is connected between the artificial zero-point created by the neutral and the line capacitors. If the substation does not have a voltage transformer VT (if the voltage of the transmission line is $35 \mathrm{kV}$ ), then the voltage of the VT type connected to the neutral of the power transformer for the signal control signal is introduced through the transformer.

As a result, it will be possible to control the operation of maintenance-free reclamation pumps that regulate the water level and the presence of mechanical impurities. The block diagram of the remote signaling of irrigation systems is shown in Figure 4. 


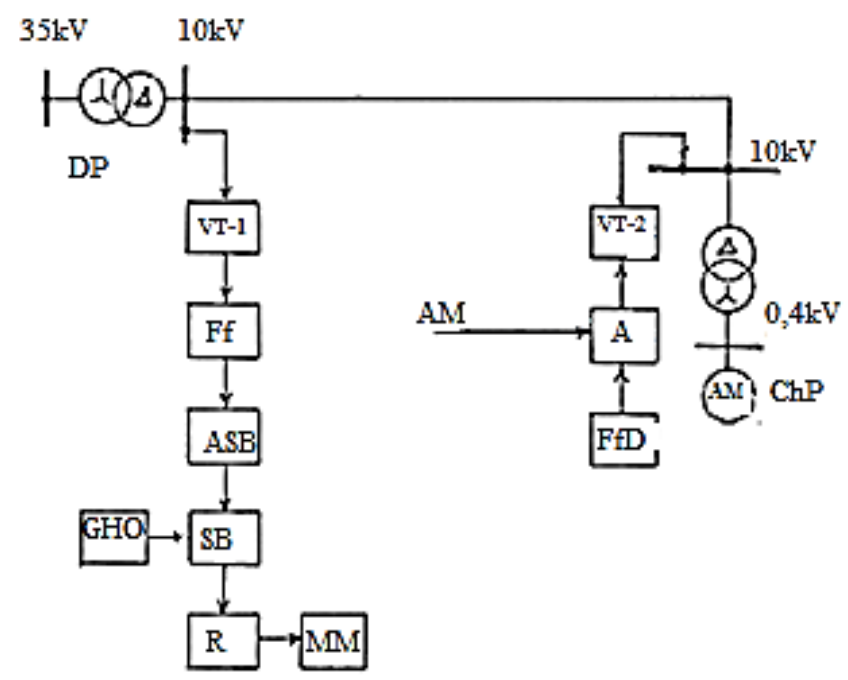

Fig .4. Block diagram of remote signaling of irrigation systems.

\section{Results and Discussion}

The proposed remote signaling system is simpler and less known [17]. Its use is economically beneficial to control the operation of pumps located within a radius of 15$20 \mathrm{~km}$ in drainage networks.

In the reclamation networks of the Bukhara region, opportunities will be created to improve the state of saline lands in agriculture through the use of experimental research and approbation of the remote signaling system developed according to the scheme shown in Figure 4.

In Figure 4, the initial ASB data are as follows:

1. The nominal values of the voltage $U_{1}$ of the nominal power supply of the ASB and the limits of the deviation of the voltage $U_{1}$ from the nominal value.

2. Frequency distribution speed;

3. Number of phases of input and output voltages;

4. $\mathrm{U}_{2}$ par nominal value of the output voltage;

5. Rated output power;

6. Load power factor;

7. Ambient temperature limits;

8. Cooling conditions;

9. Connection sequence.

The power supply of the ASB is carried out from the substation's own voltage transformer if it is present at the substation or from $10 / 0.4 \mathrm{kV}$ low voltage power transformers. Therefore, when choosing the nominal voltage consumption, ASB can be recommended voltage of $220 \mathrm{~V}$. The study of three-circuit ferroresonant circuits shows that when the input voltage changes by $+20 \%$, the output voltages at the frequency do not exceed $5 \%$.

Thus, the permissible deviations of the input voltage fluctuations in the device are taken up to $20 \%$.

When designing the ASB, two approaches to the engineering calculation method were considered: 
This is the calculation of a ferromagnetic frequency divider with the smallest weight and smallest dimensions.

Based on the provided initial data, the calculation of the ASB with the smallest dimensions can be divided into the following stages:

1. Selection of magnetic material and structure of the magnetic circuit;

2. Determine the battery capacity;

3. The core of magnetic circuits of saturated transformers ASB, calculation of dimensions;

4. Determination of the number of packages of wrappers;

5. Constructive calculation of ASB and coil weight identification;

6. Calculation of efficiency and waste;

\section{Conclusions}

Thus, based on the analysis results, ferroresonant frequency dividers were manufactured. Low harmonic frequencies were generated to create a remote signaling system using voltage transformers through high-voltage power lines.

Through the implementation of the above measures, recommendations have been developed to ensure the optimal operation of drainage pumps using harmonic frequency signals through power lines and the rational use of electricity by consumers, which leads to a decrease in energy consumption by $10-15 \%$ of total energy consumption.

\section{References}

1. Atabekov G.I., Timofeev A.V., Khukhrikov S.S. Teoretiches - Kie Foundations of Electrical Engineering. 2, Energy, (1970).

2. Bessonov A.A. Nonlinear electrical circuits. M. " High School". p 429. (1978).

3. Ismailov Z.I, Rakhimov K.R.. Subharmonic oscillations of the third order in a circuit with active nonlinear elements. IVUZ, "Radioelectronics", 1, (1972)

4. A.S. Karimov, G.R. Rakhimov, Autoparametric conversion of the number of phases of the AC frequency. Tashkent .: "Fan", p. 1975.-175

5. A.S. Karimov, M.T.Turdyev. Peculiarities of excitation of subharmonic oscillations in multicircuit ferroresonant AC circuits. 'Electricity. - eleven. pp. 20-25. (1979).

6. Karimov A.S., Burkhankhodzhaev A.M., Lvov V.K. Investigation of multicircuit autoparametric circuits in the polyharmonic mode of oscillation excitation. IVUZ. Energy. 1. Pp 32-35. (1984)

7. Karimov A.S., Ibadullaev M.I., Abdurakhmonov A.G. Ener-energetically analysis approach steady SGK second order in electromagnetic circuits. IVUZ. Energy. (6). (1988).

8. A.S.Karimov, On the theory of autoparametric frequency division in ferroresonant circuits. "Electricity", 8, (1974).

9. Karimov A.S., Lvov V.K., Radjabov I.B. Investigation of the stability of auto-parametric oscillations in a double-loop ferroresonant auto-parametric circuit. IzB. AN Uz. SSR. Ser .Teh.nauk. (5). p. 22-26. (1988).

10. Turdyev M.T, Khudaykulov W.P. Development of remote signaling systems for power transmission lines at substations without service personnel based on ferroresonant frequency converters and the number of phases, Research report, 4, (81), state. register. 1057120, Bukhara, p 22. (1984).

11. Turdyev M.T., Ibragimov N.R., Kamalov U.U. Avtopara pair metric inverters and the number of phases in the dis - petcherskogo management of distribution networks. Ed.A HUzSSR Series tehn.nauk. (3). p. 63-64. (1989). 
12. Turdyev MT, Khudoqulov Z.P Ibragimov N.R, Shoymatov BH, Quasi - sensor mode of selfoscillation in n-loop ferromagnetic converters. Abstracts "All-Union Scientific and Technical Conference." Conversion technology problems. Kiev. Institute of Electrodynamics Academy of

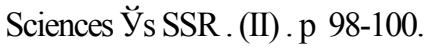

13. Turdyev M.T, Kamalov U.U, Shaymatov B.H, Schematic analysis of multi-circuit ferroresonant circuits. Sat. Topical issues of scientific works in the field of mechanics, improvement and ra $h$ vitie technological systems "Tashkent, TashP And , p 56-64.(1991).

14. Patent 11 NDR9400649 1 (Republic of Uzbekistan). Ferroresonant frequency converter. TurdyevM.T.; Burkhankhodzhaev A.M.,IbragimovN.R., Shaimatov V.Kh.-publ. 26.07.94.

15. Shaymatov B. Optimization of the process of transforming the parameters of electricity in ferroresonance circuits. Scientific-practical conference on the problems of resource and energy saving in the national economy of the Republic of Uzbekistan. Fall of scientific articles. Buxoro . p. 471-473. (1993).

16. B.Shaymatov, D.A.Rakhmatov, A.F.Mukhtorov, M.U.Rakhmatova " Probe of process of multiple - loop chains of parallel and consecutive joints, Rudenko International Conference on Methodological Problems in Reliability Study of Large Energy Systems, "Scopus" https://www.e3sconferences.org/articles/e3sconf/abs/ 2020/76/e3sconf_rses2020_01142/e3sconf_rses2020_01142.html. (2020).

17. Khafizov II, Komil Gafforov, Bakhodir Oblokulov, Aziz Azimov E limination of energy losses in pumping installations by means variable frequency drive, International Engineering Journal For Research \& Development, Vol.5, Issue 3, April 2020, E-ISSN NO: -2349-0721, Impact factor: 6.03.P.8389 , http://iejrd.com/index.php/\%20/article/view/17/5. (2020)

18. Gafurov M.O. The main energy saving measures in industrial enterprises and their effectiveness . https : // 7 universum . com / ru / tech / archive / item / 8578

19. Islom Khafizov, Komil Gafforov, Muxammedov Sh., Jurakulov A Energy saving when using a variable frequency drive in pump installations, Journal of Critical Reviews, ISSN-2394-5125 Vol 7, Issue 12, 2020, P.99102, http://dx.doi.org/10.31838/jcr.07.12.16. (2020)

20. Gafurov M.O. Mukhtorov A.F. Fattoev M.Kh. Farmonov F.A. Increasing energy efficiency through the development of energy-saving measures through energy audits in general industrial mechanisms. https://7universum.com/ru/tech/archive/item/11252 